\title{
Statistical Properties of Lorentz Gas with Periodic Configuration of Scatterers
}

\author{
L. A. Bunimovich and Ya. G. Sinai \\ Landau Institute of Theoretical Physics, Academy of Sciences of the UdSSR, Moscow, USSR
}

\begin{abstract}
In our previous paper Markov partitions for some classes of dispersed billiards were constructed. Using these partitions we estimate the decay of velocity auto-correlation function and prove the central limit theorem of probability theory and Donsker's Invariance Principle for Lorentz Gas with periodic configuration of scatterers.
\end{abstract}

\section{Introduction}

We consider in this paper the dynamical system which corresponds to the motion of a single particle between fixed scatterers on the plane $R^{2}$. Outside all scatterers the particle moves with the constant velocity and at the moments of reflections it changes its velocity according to the usual law of elastic collisions.

We assume that scatterers are disks of arbitrary diameters and the configuration of scatterers is invariant under a discrete subgroup $\Gamma$ with a compact fundamental domain of the group of all translations of the plane. The fundamental domain of $\Gamma$ can be chosen as a semi-open set the closure of which is a rectangular. We shall denote it by

$$
\Pi=\left\{q=\left(q_{1}, q_{2}\right) \mid 0 \leqq q_{1}<B_{1}, 0 \leqq q_{2}<B_{2}\right\} .
$$

Another assumption concerns the existence of a constant $A$ such that the length of any straight segment which avoids all scatterers cannot be more than $A$. Sometimes the last property is called as the property to have a finite horizon (see [1]).

The phase space $\mathscr{M}$ of our dynamical system consists of points $x=(q, v)$, where $q=\left(q^{1}, q^{2}\right)$ are coordinates, $v=\left(v^{1}, v^{2}\right)$ is velocity of the particle. Without any loss of generality we can restrict ourselves by the case $\|v\|=\sqrt{\left(v^{1}\right)^{2}+\left(v^{2}\right)^{2}}=1$. The flow corresponding to our dynamical system will be denoted by $\left\{S^{t}\right\}$. In Theorem 1 we consider a natural special representation of the flow $\left\{S^{t}\right\}$. Namely let $\mathscr{M}_{1}$ be the space of points $x=(q, v)$ such that $q$ belongs to the boundary of one of the scatterers and $v$ is directed inside the scatterers. We denote by $T_{0}$ the transformation of $\mathscr{M}_{1}$ into itself which arises when the point $x \in \mathscr{M}_{1}$ moves along its trajectory till the 
next reflection from a scatterer and $T_{0} x=\left(q_{1}, v_{1}\right)$, where $q_{1} \in R^{2}$ is the point where the next reflection takes place and $v_{1}$ is the velocity in the moment after the reflection. If $q$ is a point on the boundary of a scatterer then $n(q)$ is the unit normal vector directed outwards the scatterer and $\cos \varphi=(n(q), v),+\frac{\pi}{2}<\varphi<\frac{3 \pi}{2}$, where $(\cdot, \cdot)$ denotes the scalar product. Thus for every scatterer $\mathscr{D}_{i}^{\prime}$ we can introduce natural coordinates $r, \varphi$ on the set $\mathscr{D}_{i} \subset \mathscr{M}_{1}$ of points $x=(q, v), q \in \mathscr{D}_{i}^{\prime}$, where $r$ is a cyclic coordinate along the boundary $\mathscr{D}_{i}^{\prime}$ and $\varphi$ measures the angle between $n(q)$ and $v$. Let $d \mu_{0}$ be the differential of the measure on the set $\mathscr{D}^{(\text {per })}=\bigcup_{\mathscr{D}_{i} \subset \Pi} \mathscr{D}_{i}{ }^{1}$ such that its restriction to $\mathscr{D}_{i}$ is proportional to $|\cos \varphi| d r d \varphi$.

Theorem 1. There exists a constant $\gamma, 0<\gamma \leqq 1$, such that for all sufficiently large $n$

$$
|E(v(n), v(0))| \leqq \exp \left(-n^{\gamma}\right) .
$$

Here for $x=(q, v) \in \mathscr{D}^{(\text {per })}$ we denote $v(0)=v, T_{0}^{n} x=(q(n), v(n))$ and expectation is taken with respect to the measure $\mu_{0}$.

The proof of the Theorem 1 is contained in the Sect. 2 . Let $\mu$ be a probability measure concentrated on the set $\mathscr{M} \cap\left(\Pi x S^{1}\right)$ which is absolutely continuous with respect to the Lebesque measure on $\mathscr{M}$ and its density $p(x) \in C^{1}$. We consider $x$ as a random variable distributed according to the measure $\mu$. If $S^{t} x=x(t)=(q(t), v(t))$ then $q(t), v(t)$ are also random variables.

Theorem 2. There exists a non-degenerate two-dimensional gaussian probability distribution with the density $g$ such that

$$
\lim _{t \rightarrow \infty} \mu\left\{x: \frac{q(t)}{\sqrt{t}} \in C\right\}=\int_{C} g\left(q^{1}, q^{2}\right) d q^{1} d q^{2} .
$$

Here $C$ is a bounded open subset of the plane, the boundary of which has the area equal to zero. The next theorem is a stronger version of Theorem 2 . For every $t$ we put $q_{t}(s)=\frac{1}{\sqrt{t}} q(s t), 0 \leqq s \leqq 1$. The measure $\mu$ induces the probability distribution on the set of all possible trajectories $q_{t}(s), 0 \leqq s \leqq 1$, which are points of the space $C_{[0,1]}\left(R^{2}\right)$ of continuous functions defined on the segment $[0,1]$ with values in $R^{2}$. We shall denote this measure by $\mu_{t}$.

Theorem 3. The measures $\mu_{t}$ converge weakly to a Wiener measure.

Theorems 1-3 are derived from the properties of the Markov partition $\eta$ constructed in our previous paper [2]. The Markov partition $\eta$ is a countable partition of the phase space $\mathscr{D}^{\text {(per) }}$. Its elements are denoted by $A_{\omega_{i}}, \omega_{i} \in W$ where $W$ is the set indices.

Let $T$ be the transformation of the set $\mathscr{D}^{(\text {per })}$ induced by periodic boundary conditions. We shall list the properties of the symbolic representation of $T$ established in [2]. Let $\Omega$ be the space of sequences $\omega=\left\{\omega_{i}\right\}_{-\infty}^{\infty}$ where $\omega_{i}$ takes values in $W$. We consider the mapping $\varphi: \mathscr{D}^{(p e r)} \rightarrow \Omega$ where $\varphi(x)=\omega$ if $T^{n} x \in A_{\omega_{n}}$,

1 The notation $\mathscr{D}_{i} \subset \Pi$ means that the centrum of $\mathscr{D}_{i}^{\prime}$ belongs to $\Pi$ 
$-\infty<n<\infty$. For every $A_{\omega_{i}}$ one can define its \pm -ranks which are denoted by $r_{+}\left(A_{\omega_{i}}\right), r_{-}\left(A_{\omega_{i}}\right)$. We shall write $r_{+}\left(\omega_{n}\right), r_{-}\left(\omega_{n}\right)$ instead of $r_{+}\left(A_{\omega_{n}}\right), r_{-}\left(A_{\omega_{n}}\right)$. The measure $\varphi^{*} \mu_{0}$ is defined on the natural $\sigma$-algebra of subsets $\Omega$ and is invariant under the shift. It will be convenient to denote $\varphi^{*} \mu_{0}$ by $\mu_{0}$. In all cases it will be clear whether we consider the measure on $\mathscr{D}^{(\text {per })}$ or on $\Omega$.

Lemma 1. (see [2, Sect. 6]). There exists $\lambda_{1}, 0<\lambda_{1}<1$, such that for all sufficiently large $k$

$$
\mu_{0}\left(\omega: r_{+}\left(\omega_{0}\right) \geqq k\right) \leqq \lambda_{1}^{k}, \quad \mu_{0}\left(\omega: r_{-}\left(\omega_{0}\right) \geqq k\right) \leqq \lambda_{1}^{k}
$$

Let us introduce one-sided conditional probabilities

$$
\mu_{0}\left(\omega_{0} \mid \omega_{-1}, \omega_{-2}, \ldots, \omega_{-n}, \ldots\right)
$$

which exist with $\mu_{0}$-probability 1 . Many important properties of our dynamical system follow from the possibility of approximation of $\mu_{0}\left(\omega_{0} \mid \omega_{-1}, \ldots, \omega_{-n}, \ldots\right)$ by conditional probabilities with finite memory $\mu_{0}\left(\omega_{0} \mid \omega_{-1}, \ldots, \omega_{-n}\right)$. We shall describe the character of approximation which is valid in our case (see [2]). For any constants $\lambda_{20}, \lambda_{21}, \lambda_{22}, \lambda_{20}<\lambda_{22}, 0<\lambda_{20}, \lambda_{21}, \lambda_{22}<1$ we introduce the sets:

$$
\begin{aligned}
U_{m} & =\left\{x: \operatorname{dist}\left(x, \partial \mathscr{D}^{(\text {per })}\right)<\lambda_{20}^{m}\right\}, \quad m=1,2,3, \ldots \\
V_{n} & =\left\{x: T^{k} x \notin U_{m}, m=\left[n^{\lambda_{21}}\right],|k| \leqq n ; T^{i} x \notin U_{i} \text { for }|i|>n\right\}, \\
Z_{n} & =\left\{x: \mu_{0}\left(V_{n} \mid C_{\zeta^{-}}(x)\right)>1-\lambda_{22}^{n^{1 / 2}}\right\} .
\end{aligned}
$$

Here $\zeta^{-}$is the partition of $\Omega$ which appears when we fix all $\omega_{i},-\infty<i \leqq 0, C_{\zeta-}(x)$ is the element of $\zeta^{-}$containing $\varphi(x)$.

It is easy to see that

$$
\begin{gathered}
\mu_{0}\left(U_{m}\right)<\lambda_{20}^{m}, \mu_{0}\left(\bar{V}_{n}\right) \leqq 2 n \lambda_{20}^{\left[n^{\left.\lambda_{21}\right]}\right.}+\lambda_{20}^{n} /\left(1-\lambda_{20}\right), \\
\mu_{0}\left(\bar{Z}_{n}\right)<\frac{2 n}{1-\lambda_{20}}\left(\frac{\lambda_{20}}{\lambda_{22}}\right)^{\left[n^{\left.\lambda_{21}\right]}\right.} .
\end{gathered}
$$

Lemma 2. Let $C_{\zeta^{-}}^{\prime}, C_{\zeta^{-}}^{\prime \prime}$ correspond to $\omega_{0}, \omega_{-1}, \ldots, \omega_{-n+1}, \omega_{-n}^{\prime}, \omega_{-n-1}^{\prime}, \ldots$, and $\omega_{0}$, $\omega_{-1}, \ldots, \omega_{-n+1}, \omega_{-n}^{\prime \prime}, \omega_{-n-1}^{\prime \prime}, \ldots$ respectively and $C_{\zeta^{-}}^{\prime}, C_{\zeta_{-}}^{\prime \prime} \in \varphi\left(Z_{n}\right)$. Then one can choose $\lambda_{20}, \lambda_{21}, \lambda_{22}$, and $\lambda_{2}, 0<\lambda_{2}<1$ in such a way that

$$
\sum_{\omega_{1}}\left|\mu_{0}\left(\omega_{1} \mid C_{\zeta^{-}}^{\prime}\right)-\mu_{0}\left(\omega_{1} \mid C_{\zeta^{-}}^{\prime \prime}\right)\right| \leqq \lambda_{2}^{n^{1 / 2}}
$$

The last property is an analogy of the famous Doeblin's condition in the theory of usual countable Markov chains (see [3]). Let us consider conditional probabilities

$$
\begin{aligned}
& \pi_{1}\left(\omega_{3 n+1}, \ldots, \omega_{4 n}\right)=\mu_{0}\left(\omega_{3 n+1}, \ldots, \omega_{4 n} \mid \omega_{-n+1}^{\prime}, \ldots, \omega_{0}^{\prime}\right), \\
& \pi_{2}\left(\omega_{3 n+1}, \ldots, \omega_{4 n}\right)=\mu_{0}\left(\omega_{3 n+1}, \ldots, \omega_{4 n} \mid \omega_{-n+1}^{\prime \prime}, \ldots, \omega_{0}^{\prime \prime}\right) .
\end{aligned}
$$

We have the probability distributions $\pi_{1}, \pi_{2}$ on the space of words $\omega_{3 n+1}, \ldots, \omega_{4 n}$ under different conditions $\omega_{-n+1}^{\prime}, \ldots, \omega_{0}^{\prime}$ and $\omega_{-n+1}^{\prime \prime}, \ldots, \omega_{0}^{\prime \prime}$. 
Lemma 3. Suppose that $r_{ \pm}\left(\omega_{i}^{\prime}\right), r_{ \pm}\left(\omega_{i}^{\prime \prime}\right) \leqq n, 1 \leqq i \leqq n$. There exists a constant $\lambda_{3}$, $0<\lambda_{3}<1$ such that for all large enough $n$

$$
\operatorname{Var}\left(\pi_{1}, \pi_{2}\right)=\frac{1}{2} \sum_{\omega_{3 n+1}, \ldots, \omega_{4 n}}\left|\pi_{1}\left(\omega_{3 n+1}, \ldots, \omega_{4 n}\right)-\pi_{2}\left(\omega_{3 n+1}, \ldots, \omega_{4 n}\right)\right| \leqq \lambda_{3} .
$$

Only the properties of $\mu_{0}$ which are presented in Lemmas 1-3 will be used during the proof of our Theorems.

\section{Proof of Theorem 1}

Let $h(\omega)$ be a function defined on $\Omega$ such that $|h(\omega)| \leqq C_{1}$ where $C_{1}$ is a constant and there exists a constant $\lambda_{4}, 0<\lambda_{4}<1$, such that for all sufficiently large $n$ one can find functions $h_{n}(\omega)=h_{n}\left(\omega_{-n}, \ldots, \omega_{n}\right), \int h_{n} d \mu_{0}=0$, depending only on coordinates $\omega_{i},|i| \leqq n$ and $\sup _{\omega}\left|h(\omega)-h_{n}(\omega)\right| \leqq \lambda_{4}^{n}$. We denote also by $T_{0}$ the shift in the space $\Omega$.

Lemma 4. Let the measure $\mu_{0}$ satisfy the assertions of Lemmas 1-3. If Eh=0 then $\left|E h\left(T_{0}^{n} \omega\right) h(\omega)\right| \leqq \exp \left(-n^{\gamma}\right)$ for all sufficiently large $n$ and some constant $\gamma, 0<\gamma<1$.

Proof. Let $n$ and $n_{1}<n$ be chosen. We have

$$
\left|E h\left(T_{0}^{n} \omega\right) \cdot h(\omega)-E h_{n_{1}}\left(T_{0}^{n} \omega\right) h_{n_{1}}(\omega)\right| \leqq C_{2} \lambda_{4}^{n_{1}},
$$

where $C_{2}$ is a positive constant. We can write

$E h_{n_{1}}\left(T_{0}^{n} \omega\right) h_{n_{1}}(\omega)=\sum_{\omega-n_{1}, \ldots, \omega_{n+n_{1}}} h_{n_{1}}\left(\omega_{-n_{1}} \ldots \omega_{n_{1}}\right) h_{n_{1}}\left(\omega_{n-n_{1}} \ldots \omega_{n+n_{1}}\right) \mu_{0}\left(\omega_{-n_{1}} \ldots \omega_{n+n_{1}}\right)$.

Now we shall transform the probability distribution $\mu_{0}\left(\omega_{-n_{1}}, \ldots, \omega_{n+n_{1}}\right)$. Let $A_{n}$ be the set of words $\left(\omega_{-n_{1}}, \ldots, \omega_{n+n_{1}}\right)$ such that $\operatorname{rg}_{ \pm}\left(\omega_{i}\right) \leqq 2 n_{1}+1,-n \leqq i \leqq n+n_{1}$ and

$$
\mu_{0}\left(V_{n_{1}} \cap\left(\omega_{k} \ldots \omega_{l}\right)\right) \geqq\left(1-\sqrt{\mu_{0}\left(\bar{V}_{n_{1}}\right)}\right) \mu_{0}\left(\omega_{k} \ldots \omega_{l}\right)
$$

where $\bar{V}_{n_{1}}=M-V_{n_{1}},-n_{1} \leqq k, l \leqq n+n_{1}$. An easy application of Chebyshev's inequality shows that $\mu_{0}\left(A_{n}\right) \geqq 1-n^{2} \sqrt{\mu_{0}\left(\bar{V}_{n_{1}}\right)}$.

Lemma 4.1. For $\left(\omega_{-n_{1}} \ldots \omega_{n+n_{1}}\right) \in A_{n}$

for some $\lambda_{4}, 0<\lambda_{4}<1$.

$$
\begin{aligned}
\left(1-\sqrt{\mu_{0}\left(\bar{V}_{n_{1}}\right)}\right)^{2 n}\left(1+\lambda_{4}^{n_{1}}\right)^{-n} & \leqq \frac{\mu_{0}\left(\omega_{-n_{1}} \ldots \omega_{n+n_{1}}\right)}{\mu_{0}\left(\omega_{-n_{1}} \ldots \omega_{n_{1}}\right) \prod_{i=n_{1}+1}^{n+n_{1}} \mu_{0}\left(\omega_{i} \mid \omega_{i-1} \ldots \omega_{i-2 n_{1}-1}\right)} \\
& \leqq\left(1+\lambda_{4}^{n_{1}}\right)^{n}\left(1-\sqrt{\mu_{0}\left(\bar{V}_{n_{1}}\right)}\right)^{-2 n}
\end{aligned}
$$

Proof. We have

$$
\mu_{0}\left(\omega_{-n_{1}} \ldots \omega_{n+n_{1}}\right)=\mu_{0}\left(\omega_{-n_{1}} \ldots \omega_{n_{1}}\right) \prod_{i=n_{1}+1}^{n+n_{1}} \mu_{0}\left(\omega_{i} \mid \omega_{i-1} \ldots \omega_{-n_{1}}\right) .
$$

We shall estimate the fraction

$$
I_{i}=\frac{\mu_{0}\left(\omega_{i} \mid \omega_{i-1} \ldots \omega_{i-2 n_{1}-1}\right)}{\mu_{0}\left(\omega_{i} \mid \omega_{i-1} \ldots \omega_{-n_{1}}\right)} .
$$


We can consider also the fraction

$$
I_{i}^{(0)}=\frac{\mu_{0}\left(V_{n_{1}} \cap \omega_{i} \mid \omega_{i-1} \ldots \omega_{i-2 n_{1}-1}\right)}{\mu_{0}\left(V_{n_{1}} \cap \omega_{i} \mid \omega_{i-1} \ldots \omega_{-n_{1}}\right)}
$$

For words $\left(\omega_{-n_{1}} \ldots \omega_{n+n_{1}}\right) \in A_{n}$ we have

$$
\left(1-\sqrt{\mu_{0}\left(\bar{V}_{n_{1}}\right)}\right)^{2} \leqq I_{i}^{(0)}: I_{i} \leqq\left(1-\sqrt{\mu_{0}\left(\bar{V}_{n_{1}}\right)}\right)^{-2} .
$$

Therefore it is sufficient to estimate $I_{i}^{(0)}$. We can write

$$
I_{i}^{(0)}=\frac{\int \mu_{0}\left(V_{n_{1}} \cap \omega_{i} \mid C_{T^{i-1 \zeta^{-}}}\right) d v^{(1)}}{\int \mu_{0}\left(V_{n_{1}} \cap \omega_{i} \mid C_{T^{i-1 \zeta^{-}}}\right) d v^{(2)}},
$$

where $v^{(1)}\left(v^{(2)}\right)$ is the induced probability distribution on the space of

$$
C_{T^{i-1} \zeta^{-}} \subset\left(\omega_{i-1}, \ldots, \omega_{-n_{1}}\right)\left(C_{T^{i-1} \zeta^{-}} \subset\left(\omega_{i-1}, \ldots, \omega_{i-2 n_{1}-1}\right)\right) .
$$

Let us fix

$$
C_{T^{i-1 \zeta^{-}}}^{(0)} \in\left(\omega_{i-1}, \ldots, \omega_{-n_{1}}\right) \subset\left(\omega_{i-1}, \ldots, \omega_{i-2 n_{1}-1}\right)
$$

and rewrite $I_{i}^{(0)}$ as follows

$$
I_{i}^{(0)}=\frac{\int \frac{\mu_{0}\left(\omega_{i} \cap V_{n_{1}} \mid C_{T^{i-1 \zeta^{-}}}\right)}{\mu_{0}\left(\omega_{i} \cap V_{n_{1}} \mid C_{T^{i-1 \zeta^{-}}}^{(0)}\right)} d v^{(1)}}{\int \frac{\mu_{0}\left(\omega_{i} \cap V_{n_{1}} \mid C_{T^{1-1} \zeta^{-}}\right)}{\mu_{0}\left(\omega_{i} \cap V_{n_{1}} \mid C_{T^{i-1 \zeta^{-}}}^{(0)}\right)} d v^{(2)}} .
$$

The sets $\left(\omega_{i} \cap V_{n_{1}}\right) \cap C_{T^{i-1 \zeta^{-}}}$and $\left(\omega_{i} \cap V_{n_{1}}\right) \cap C_{T^{-1} \zeta^{-}}^{(0)}$ are canonically isomorphic for arbitrary $C_{T^{x^{-1}} \zeta^{-}}, C_{T^{1-1} \zeta^{-}}^{(0)}$ (see [2]). The absolute value of the difference between the corresponding density and 1 is not more than $\lambda_{4}^{n_{1}}$ for some $\lambda_{4}, 0<\lambda_{4}<1$. Therefore

$$
\left|\frac{\mu_{0}\left(\omega_{i} \cap V_{n_{1}} \mid C_{T^{i^{-1} \zeta^{-}}}\right)}{\mu_{0}\left(\omega_{i} \cap V_{n_{1}} \mid C_{T^{1-1} \zeta^{-}}^{(0)}\right)}-1\right| \leqq \lambda_{4}^{n_{1}}
$$

and $\left(1+\lambda_{4}^{n_{1}}\right)^{-2} \leqq I_{i}^{(0)} \leqq\left(1+\lambda_{4}^{n_{1}}\right)^{2}$. Now we have

$$
\begin{aligned}
& \frac{\mu_{0}\left(\omega_{-n_{1}} \ldots \omega_{n+n_{1}}\right)}{\mu_{0}\left(\omega_{-n_{1}} \ldots \omega_{n_{1}}\right) \prod_{i=n_{1}+1}^{n+n_{1}} \mu_{0}\left(\omega_{i} \mid \omega_{i-1} \ldots \omega_{i-2 n_{1}-1}\right)}=\prod_{i=n_{1}+1}^{n+n_{1}} I_{i} \leqq\left(1-\sqrt{\mu_{0}\left(\bar{V}_{n_{1}}\right)}\right)^{-2 n} \prod I_{i}^{(0)} \\
& \leqq\left(1+\lambda_{4}^{n_{1}}\right)^{n}\left(1-\sqrt{\mu_{0}\left(\bar{V}_{n_{1}}\right)}\right)^{-2 n} .
\end{aligned}
$$

In an analogous way one can get easily a similar estimation from below. Q.E.D.

It follows easily from the Lemma 4.1 that the problem is reduced to the investigation of the expression

$$
\begin{aligned}
& \sum h_{n_{1}}\left(\omega_{-n_{1}} \ldots \omega_{n_{1}}\right) h_{n_{1}}\left(\omega_{n-n_{1}} \ldots \omega_{n+n_{1}}\right) \mu_{0}\left(\omega_{-n_{1}} \ldots \omega_{n_{1}}\right) \\
& \quad \prod_{i=n_{1}+1}^{n+n_{1}} \mu_{0}\left(\omega_{i} \mid \omega_{i-1} \ldots \omega_{i-2 n_{1}-1}\right) .
\end{aligned}
$$

The numbers

$$
\mu_{0}^{(1)}\left(\omega_{-n_{1}} \ldots \omega_{n+n_{1}}\right)=\mu_{0}\left(\omega_{-n_{1}} \ldots \omega_{n_{1}}\right) \prod_{i=n_{1}+1}^{n+n_{1}} \mu_{0}\left(\omega_{i} \mid \omega_{i-1} \ldots \omega_{i-2 n_{1}-1}\right)
$$


define the probability distribution on the space of all words $\omega_{-n_{1}} \ldots \omega_{n+n_{1}}$ which is homogeneous Markov chain with the memory $2 n_{1}+1$. Its stationary probabilities are equal to $\mu_{0}\left(\omega_{-n_{1}} \ldots \omega_{n_{1}}\right)$ while the transition probabilities have the form $\mu_{0}\left(\omega_{i} \mid \omega_{i-1} \ldots \omega_{i-2 n_{1}-1}\right)$. The space of states of the Markov chain consists of all words $\left(\omega_{-n_{1}} \ldots \omega_{n_{1}}\right)$. Let us introduce the subset $\mathscr{E}_{n_{1}}$ of the space of states which is defined via the conditions:

1. $\operatorname{rg}_{ \pm}\left(\omega_{i}\right) \leqq 2 n_{1}+1,-n_{1} \leqq i \leqq n_{1}$;

2. $\mu_{0}\left(\left(\omega_{-n_{1}} \ldots \omega_{n_{1}}\right) \cap V_{n_{1}}\right) \geqq\left(1-\sqrt{\mu_{0}\left(\bar{V}_{n_{1}}\right)}\right) \mu_{0}\left(\omega_{-n_{1}} \ldots \omega_{n_{1}}\right)$;

3. a transition $\left(\omega_{-n_{1}} \ldots \omega_{n_{1}}\right) \rightarrow\left(\omega_{-n_{1}+1} \ldots \omega_{n_{1}+1}\right)$ will be called admissible if

$$
\mu_{0}\left(\left(\omega_{-n_{1}} \ldots \omega_{n_{1}+1}\right) \cap V_{n_{1}}\right) \geqq\left(1-\sqrt{\mu_{0}\left(\bar{V}_{n_{1}}\right)}\right) \mu_{0}\left(\left(\omega_{-n_{1}} \ldots \omega_{n_{1}+1}\right)\right) ;
$$

by definition for all states $\left(\omega_{-n_{1}} \ldots \omega_{n_{1}}\right) \in \mathscr{E}_{n_{1}}$ the conditional probability of admissible transitions is not less than $\sqrt{\mu_{0}\left(V_{n_{1}}\right)}$.

We introduce the subset $\mathscr{B}_{n}$ of all words $\left(\omega_{-n_{1}} \ldots \omega_{n+n_{1}}\right)$ for which $\left(\omega_{i-n_{1}} \ldots \omega_{i+n_{1}}\right) \in \mathscr{E}_{n}, 0 \leqq i \leqq n$, and all transitions

$$
\left(\omega_{i-n_{1}} \ldots \omega_{i+n_{1}}\right) \rightarrow\left(\omega_{i+1-n_{1}} \ldots \omega_{i+1+n_{1}}\right)
$$

are admissible. We define a new probability distribution $\mu_{0}^{(1)}$ on the space $\mathscr{B}_{n}$ by putting for $\left(\omega_{-n_{1}} \ldots \omega_{n+n_{1}}\right) \in B_{n}$

$$
\mu_{0}^{(1)}\left(\omega_{-n_{1}} \ldots \omega_{n_{1}+n}\right)=Z^{-1} \mu_{0}\left(\omega_{-n_{1}} \ldots \omega_{n_{1}}\right) \prod_{i=n_{1}+1}^{n+n_{1}} \mu_{0}\left(\omega_{i} \mid \omega_{i-1} \ldots \omega_{i-2 n_{1}-1}\right)
$$

where $Z$ is a partition function,

$$
Z=\sum_{\omega_{-n_{1}} \ldots \omega_{n+n_{1}}} \mu_{0}\left(\omega_{-n_{1}} \ldots \omega_{n_{1}}\right) \prod_{i=n_{1}+1}^{n+n_{1}} \mu_{0}\left(\omega_{i} \mid \omega_{i-1} \ldots \omega_{i-2 n_{1}-1}\right) .
$$

An easy application of Chebyshev's inequality shows that

$$
\mu_{0}\left(B_{n}\right) \geqq 1-2\left(n+2 n_{1}+1\right) \sqrt{\mu_{0}\left(\bar{V}_{n_{1}}\right)}-\left(2 n_{1}+n+1\right) \lambda_{1}^{2 n_{1}+1} .
$$

It follows from Lemma 4.1 .

$$
e^{-\varepsilon_{n}} \leqq \frac{\mu_{0}^{(1)}\left(\omega_{-n_{1}} \ldots \omega_{n+n_{1}}\right)}{\mu_{0}\left(\omega_{-n_{1}} \ldots \omega_{n+n_{1}}\right)} \leqq e^{\varepsilon_{n}}
$$

for

$$
\left(\omega_{-n_{1}} \ldots \omega_{n+n_{1}}\right) \in A_{n} \cap B_{n}, \quad \varepsilon_{n}=n \ln \left(1+\lambda_{4}^{n_{1}}\right)-2 n \ln \left(1-\mu_{0}\left(\bar{V}_{n_{1}}\right)\right) .
$$

As a result we get an estimation $Z \geqq \mu_{0}\left(V_{n_{1}}\right) \exp \left(-\varepsilon_{n}\right)$ which shows that the probability distributions $\mu_{0}^{(1)}$ and $\mu_{0}$ are very close to each other on the set $A_{n} \cap B_{n}$. It shows also that it is sufficient for our aims to consider the expression

$$
\begin{aligned}
b_{n}= & \sum \mu_{0}^{(1)}\left(\omega_{-n_{1}} \ldots \omega_{n+n_{1}}\right) h_{n_{1}}\left(\omega_{-n_{1}} \ldots \omega_{n_{1}}\right) h_{n_{1}}\left(\omega_{n-n_{1}} \ldots \omega_{n+n_{1}}\right) \\
= & \sum \mu_{0}^{(1)}\left(\omega_{-n_{1}} \ldots \omega_{n_{1}}\right) h_{n_{1}}\left(\omega_{-n_{1}} \ldots \omega_{n_{1}}\right) \\
& \cdot \mu_{0}^{(1)}\left(\omega_{n-n_{1}} \ldots \omega_{n+n_{1}} \mid \omega_{-n_{1}} \ldots \omega_{n_{1}}\right) h_{n_{1}}\left(\omega_{n-n_{1}} \ldots \omega_{n+n_{1}}\right) .
\end{aligned}
$$


The probability distribution $\mu_{0}^{(1)}$ is a non-homogeneous Markov chain of the memory $2 n_{1}+1$. Its transition probabilities have the form

$$
=\frac{\mu_{0}\left(\omega_{i} \mid \omega_{i-1} \ldots \omega_{i-2 n_{1}-1}\right) \sum_{\omega_{i+1} \ldots \omega_{n}} \prod_{j=i+1}^{(1)}\left(\omega_{i} \mid \omega_{i-1} \ldots \omega_{i-2 n_{1}-1}\right)}{\sum_{\omega_{i} \ldots \omega_{n+n_{1}}} \prod_{j=i}^{n+n_{1}} \mu_{0}\left(\omega_{j} \mid \omega_{j-1} \ldots \omega_{j-1} \ldots \omega_{j-2 n_{1}-1}\right)} .
$$

In both cases the sums are taken over such words that $\left(\omega_{j-2 n_{1}} \ldots \omega_{j}\right) \in \mathscr{E}_{n}$ and all transitions are admissible. It follows easily from the properties of $\mathscr{E}_{n}$ that these sums are very close to one. Namely, the absolute values of their differences from 1 are not more than $\left(1-\sqrt{\mu_{0}\left(\bar{V}_{n_{1}}\right)}\right)^{n+2 n_{1}+1}$. Thus

$$
\left|\frac{\mu_{0}^{(1)}\left(\omega_{i} \mid \omega_{i-1} \ldots \omega_{i-2 n_{1}-1}\right)}{\mu_{0}\left(\omega_{i} \mid \omega_{i-1} \ldots \omega_{i-2 n_{1}-1}\right)}-1\right| \leqq 1-\left(\sqrt{\mu_{0}\left(\bar{V}_{n_{1}}\right)}\right)^{2\left(n+2 n_{1}+1\right)} .
$$

The same arguments show that for any $m$ the variation

$$
\begin{aligned}
& \frac{1}{2} \sum \mid \mu_{0}^{(1)}\left(\omega_{3\left(2 n_{1}+1\right)+m+1} \ldots \omega_{4\left(2 n_{1}+1\right)+m} \mid \omega_{-n_{1}}^{\prime} \ldots \omega_{n_{1}}^{\prime}\right) \\
& \quad-\mu_{0}^{(1)}\left(\omega_{3\left(2 n_{1}+1\right)+m+1} \ldots \omega_{4\left(2 n_{1}+1\right)+m} \mid \omega_{-n_{1}}^{\prime \prime} \ldots \omega_{n_{1}}^{\prime \prime}\right) \mid
\end{aligned}
$$

differs from the analogous variation for $\mu_{0}$ to a number whose absolute value is not more than $2\left|1-\left(1-\sqrt{\mu_{0}\left(\bar{V}_{n_{1}}\right)}\right)^{4\left(2 n_{1}+1\right)}\right|$. Now from Lemma 3 we get that the last sum is not more than $\lambda_{3}^{\prime}$ where $0<\lambda_{3}^{\prime}<1$ and does not depend on $n$. Therefore from the usual ergodic theorem for Markov chains

$$
\begin{aligned}
& \frac{1}{2} \sum\left|\mu_{0}^{(1)}\left(\omega_{n-n_{1}} \ldots \omega_{n+n_{1}} \mid \omega_{-n_{1}}^{\prime} \ldots \omega_{n_{1}}^{\prime}\right)-\mu_{0}^{(1)}\left(\omega_{n-n_{1}} \ldots \omega_{n+n_{1}} \mid \omega_{-n_{1}}^{\prime \prime} \ldots \omega_{n_{1}}^{\prime \prime}\right)\right| \\
& \quad \leqq \operatorname{Const}\left(\lambda_{3}^{\prime}\right)^{\frac{n}{3\left(2 n_{1}+1\right)}} .
\end{aligned}
$$

Let us fix $\omega_{-n_{1}}^{(0)} \ldots \omega_{n_{1}}^{(0)}$. We have

$$
\begin{aligned}
b_{n}= & \left(\sum \mu_{0}^{(1)}\left(\omega_{-n_{1}} \ldots \omega_{n_{1}}\right) h_{n_{1}}\left(\omega_{-n_{1}} \ldots \omega_{n_{1}}\right)\right) \sum h_{n_{1}}\left(\omega_{n-n_{1}} \ldots \omega_{n+n_{1}}\right) \\
& \cdot \mu_{0}^{(1)}\left(\omega_{n-n_{1}} \ldots \omega_{n+n_{1}} \mid \omega_{-n_{1}}^{(0)} \ldots \omega_{n_{1}}^{(0)}\right) \\
& +\sum \mu_{0}^{(1)}\left(\omega_{-n_{1}} \ldots \omega_{n_{1}}\right) h_{n_{1}}\left(\omega_{-n_{1}} \ldots \omega_{n_{1}}\right) \\
& \cdot\left(\sum h _ { n _ { 1 } } ( \omega _ { n - n _ { 1 } } \ldots \omega _ { n + n _ { 1 } } ) \left(\mu_{0}^{(1)}\left(\omega_{n-n_{1}} \ldots \omega_{n+n_{1}} \mid \omega_{-n_{1}} \ldots \omega_{n_{1}}\right)\right.\right. \\
& \left.-\mu_{0}^{(1)}\left(\omega_{n-n_{1}} \ldots \omega_{n+n_{1}} \mid \omega_{-n_{1}}^{(0)} \ldots \omega_{n_{1}}^{(0)}\right)\right) .
\end{aligned}
$$

The absolute value of the second term is less than $\operatorname{const}\left(\lambda_{3}^{\prime}\right)^{\frac{n}{3\left(2 n_{1}+1\right)}}$. Concerning the first term we can write

$$
\begin{gathered}
\sum \mu_{0}^{(1)}\left(\omega_{-n_{1}} \ldots \omega_{n_{1}}\right) h_{n_{1}}\left(\omega_{-n_{1}} \ldots \omega_{n_{1}}\right) \\
=\sum\left(\mu_{0}^{(1)}\left(\omega_{-n_{1}} \ldots \omega_{n_{1}}\right)-\mu_{0}\left(\omega_{-n_{1}} \ldots \omega_{n_{1}}\right)\right) h_{n_{1}}\left(\omega_{-n_{1}} \ldots \omega_{n_{1}}\right) \\
=\sum \mu_{0}\left(\omega_{-n_{1}} \ldots \omega_{n_{1}}\right)\left(\frac{\mu_{0}^{(1)}\left(\omega_{-n_{1}} \ldots \omega_{n_{1}}\right)}{\mu_{0}\left(\omega_{-n_{1}} \ldots \omega_{n_{1}}\right)}-1\right) h_{n_{1}}\left(\omega_{-n_{1}} \ldots \omega_{n_{1}}\right) .
\end{gathered}
$$


The absolute value of the last expression is not more than

$$
\left[1-\left(1-\sqrt{\mu_{0}\left(\bar{V}_{n_{1}}\right)}\right)^{2\left(n+2 n_{1}+1\right)}\right] \max \left|h_{n_{1}}\right| \text {. Q.E.D. }
$$

Theorem 1 follows immediately from Lemma 4. It follows easily from Theorem 1 that for considered $h E\left(\sum_{0 \leqq i \leqq k} h\left(T^{i} x\right)\right)^{2}$
$\sim$ const $k$ as $k \rightarrow \infty$, where const depends only on $h$.

Theorem 1'. For the same class of functions $h$

$$
E\left(\sum_{0 \leqq i \leqq k} h\left(T^{i} x\right)\right)^{4} \sim \text { const } k^{2}
$$

Proof. Let $n_{1}=\left[n^{\alpha_{1}}\right]$ where $\alpha_{1}$ is small enough. It is sufficient to estimate

$$
E\left(\sum_{0 \leqq i \leqq k} h_{n_{1}}\left(T^{i} x\right)\right)^{4}=\sum E h_{n_{1}}\left(T^{i_{1}} x\right) h_{n_{1}}\left(T^{i_{2}} x\right) h_{n_{1}}\left(T^{i_{3}} x\right) h_{n_{1}}\left(T^{i_{4}} x\right)
$$

We shall estimate expectations

$$
E h_{n_{1}}\left(T^{i_{1}} x\right) h_{n_{1}}\left(T^{i_{2}} x\right) h_{n_{1}}\left(T^{i_{3}} x\right) h_{n_{1}}\left(T^{i_{4}} x\right)
$$

assuming that $i_{1} \leqq i_{2} \leqq i_{3} \leqq i_{4}$. Also we can assume that

$$
m=\max \left(\left(i_{2}-i_{1}\right),\left(i_{3}-i_{2}\right),\left(i_{4}-i_{3}\right)\right) \geqq\left[n_{1}^{1+\alpha_{2}}\right], \quad \alpha_{2}>0
$$

because the total number of terms not satisfying this conditions is less than const $n^{1+3 \alpha_{1}\left(1+\alpha_{2}\right)}<n^{2}$ if $\alpha_{1}$ is small enough. The next approximation consists of replacing $\mu_{0}$ by $\mu_{0}^{(1)}$ and considering

$$
\begin{aligned}
& E^{(1)} h_{n_{1}}\left(T^{i_{1}} x\right) h_{n_{1}}\left(T^{i_{2}} x\right) h_{n_{1}}\left(T^{i_{3}} x\right) h_{n_{1}}\left(T^{i_{4}} x\right) \\
& =\sum h_{n_{1}}\left(\omega_{i_{1}-n_{1}} \ldots \omega_{i_{1}+n_{1}}\right) h_{n_{1}}\left(\omega_{i_{2}-n_{1}} \ldots \omega_{i_{2}+n_{1}}\right) h_{n_{1}}\left(\omega_{i_{3}-n_{1}} \ldots \omega_{i_{3}+n_{1}}\right) \\
& \quad \cdot h_{n_{1}}\left(\omega_{i_{4}-n_{1}} \ldots \omega_{i_{4}+n_{1}}\right) \mu_{0}^{(1)}\left(\omega_{-n_{1}} \ldots \omega_{n+n_{1}}\right)
\end{aligned}
$$

because the error also is sufficiently small. Now we consider three cases.

1. $m=i_{4}-i_{3}$. In this case we estimate the conditional expectations of $h_{n_{1}}\left(T^{i_{4}} x\right)$ under fixed $\omega_{-n_{1}}, \ldots, \omega_{i_{3}+n_{1}}$. The same arguments as above show that its absolute value is not more than const $\left(\lambda_{3}^{\prime}\right)^{m / n_{1}}$. Therefore the absolute value of the whole expectation is not more const $\left(\lambda_{3}^{\prime}\right)^{m / n_{1}}$ and the total amount of such terms is not more than const $\mathrm{nm}^{2}$. Thus the absolute value of the sum over such terms is not more than const $n \sum_{m \geqq n_{1}^{\left(1+\alpha_{2}\right)}} m^{2}\left(\lambda_{3}^{\prime}\right)^{m / n_{1}} \sim 0\left(n^{2}\right)$ for $n \rightarrow \infty$.

2. $m=i_{3}-i_{2}$. In this case we fix $\omega_{-n_{1}} \ldots \omega_{i_{2}+n_{1}}$ and consider the conditional expectation of $h_{n_{1}}\left(T^{i_{3}} x\right) h_{n_{1}}\left(T^{i_{4}} x\right)$.

The absolute value of the difference between it and the unconditional one is less than const $\left(\lambda_{3}^{\prime}\right)^{m / n_{1}}$. From the other side we have shown during the proof of Lemma 4 that the unconditional expectation of $h_{n_{1}}\left(T^{i_{3}} x\right) h_{n_{1}}\left(T^{i_{4}} x\right)$ with respect to $\mu_{0}^{(1)}$ decays very quickly and in particular is less than const $\left(i_{4}-i_{3}\right)^{-2}$. The summation of all estimates gives the desired result.

3. $m=i_{2}-i_{1}$. This case can be treated in the same way as 2. Q.E.D. 


\section{Proof of Theorem 2}

We start with proving a central limit theorem of probability theory for a function $h(\omega)$ satisfying to the same conditions as in the beginning of Sect. 2. From Theorem 1 it follows that

and consequently

$$
\sum_{n=0}^{\infty}\left|E\left(T_{0}^{n} h \cdot h\right)\right|<\infty
$$

$$
\sigma(h)=\sum_{n=0}^{\infty} E\left(T_{0}^{n} h \cdot h\right)<\infty
$$

where the expectation $E$ is taken with respect to the measure $\mu_{0}$. Suppose that $\sigma(h)>0$.

Lemma 5. Let $E(h)=0$. Then for every $a, b, a<b$ we have

$$
\lim _{n \rightarrow \infty} \mu_{0}\left(\omega: a<\frac{1}{\sqrt{\sigma_{h} n}} \sum_{k=0}^{n-1} h\left(T^{k} \omega\right)<b\right)=\frac{1}{\sqrt{2 \pi}} \int_{a}^{b} \exp \left(-\frac{u^{2}}{2}\right) d u .
$$

Proof. The statement of lemma means that the probability distribution of the normed sums $\frac{1}{\sqrt{\sigma_{n} n}} \sum_{k=0}^{n-1} h\left(T^{k} \omega\right)$ converges weakly to the standard gaussian probability distribution. The machinery of proving such theorems is now sufficiently far developed and we shall use one of the usual ways.

Let us introduce a characteristic function

$$
\varphi_{n}(\lambda)=E \exp \left(i \frac{\lambda}{\sqrt{\sigma_{h} n}} \sum_{k=0}^{n-1} h\left(T^{k} \omega\right)\right) .
$$

In order to prove lemma we must show that $\varphi_{n}(\lambda) \rightarrow \exp \left(-\frac{\lambda^{2}}{2}\right)$ when $\lambda$ takes values from any compact set and $n \rightarrow \infty$. Let us decompose the whole interval $[0, n-1]$ onto non-overlapping subintervals

$$
\Delta=\Delta_{1}^{(1)} \cup \Delta_{1}^{(2)} \cup \Delta_{2}^{(1)} \cup \Delta_{2}^{(2)} \cup \ldots \cup \Delta_{p-1}^{(2)} \cup \Delta_{p}^{(1)}
$$

in such a way that the length $\left|\Delta_{i}^{(1)}\right|$ of each $\Delta_{i}^{(1)}$ except the last one is equal to $\left[n^{\gamma_{1}}\right]$ while the length of each $\Delta_{j}^{(2)}$ is equal to $\left[n^{\gamma_{2}}\right]$, where $\gamma_{1}, \gamma_{2}>0, \frac{1}{2}+\gamma_{2}<\gamma_{1}<1$ and $\left|4_{p}^{(1)}\right| \leqq\left[n^{\gamma_{1}}\right]$. We can write now

$$
\varphi_{n}(\lambda)=E \exp \left(i \frac{\lambda}{\sqrt{\sigma_{h} n}}\left[\sum_{s=1}^{p} \sum_{k \in \Delta \xi^{1)}} h\left(T^{k} \omega\right)+\sum_{s=1}^{p-1} \sum_{\left.k \in \Delta s^{2}\right)} h\left(T^{k} \omega\right)\right]\right) .
$$

We have

$$
\left|\sum_{s=1}^{p-1} \sum_{k \in \Delta \xi^{2)}} h\left(T^{k} \omega\right)\right| \leqq \operatorname{const} p \cdot n^{\gamma_{2}} \leqq \text { const } n^{1-\gamma_{1}+\gamma_{2}}
$$

From our assumptions concerning $\gamma_{1}, \gamma_{2}$ it follows that

$$
\sup \left|\frac{1}{\sqrt{n}} \sum_{s=1}^{p-1} \sum_{k \in \Delta s^{2)}} h\left(T^{k} \omega\right)\right| \rightarrow 0
$$


when $n \rightarrow \infty$ and the limit behaviour of $\varphi_{n}(\lambda)$ is just the same as the limit behaviour of

$$
\psi_{n}^{(1)}(\lambda)=E \exp \left\{i \frac{\lambda}{\sqrt{\sigma_{h} n}} \sum_{s=1}^{p} \sum_{k \in \Delta \xi^{(1)}} h\left(T^{k} \omega\right)\right\} .
$$

We shall show that $\psi_{n}^{(1)}(\lambda)$ is close to

$$
\left[E \exp i \frac{\lambda}{\sqrt{\sigma_{h} h}} \sum_{k \in \Delta_{i}^{(1)}} h\left(T^{k} \omega\right)\right]^{p}
$$

i.e. $\psi_{n}^{(1)}(\lambda)$ is close to the characteristic function of the normed sum of $p$ independent random variables distributed as the random variable $\sum_{k \in \Lambda_{1}^{(1)}} h\left(T^{k} \omega\right)$. We choose $0<\gamma_{3}<\gamma_{2}$ and $n_{1}=\left[n^{\gamma_{3}}\right]$ and the function $h_{n_{1}}(\omega)$ (see the beginning of Sect. 2). We have

$$
\left|\sum_{s=1}^{p} \sum_{k \in \Delta \varsigma^{1)}}\left(h\left(T^{k} \omega\right)-h_{n_{1}}\left(T^{k} \omega\right)\right)\right| \leqq \operatorname{const} n^{2} \lambda_{4}^{n_{1}} .
$$

Therefore we can replace $\psi_{n}^{(1)}(\lambda)$ by

$$
\psi_{n}^{(2)}(\lambda)=E \exp \left\{i \frac{\lambda}{\sqrt{\sigma_{h} n}} \sum_{s=1}^{p} \sum_{k \in \Delta s^{(1)}} h_{n_{1}}\left(T^{k} \omega\right)\right\} .
$$

The function $\exp \left\{i \frac{\lambda}{\sqrt{\sigma_{h} n}} \sum_{s=1}^{p} \sum_{k \in \Delta \Delta^{(1)}} h_{n_{1}}\left(T^{k} \omega\right)\right\}$ depend only on variables $\omega_{-n_{1}}, \ldots, \omega_{n+n_{1}}$. Let us restrict ourselves in the integral which gives $\psi_{n}^{(2)}(\lambda)$ only by sequences for which $r_{ \pm}\left(\omega_{i}\right) \leqq 2 n_{1}+1=n_{2}$.

According to Lemma 1 the probability of these sequences is not less than $\left(1-\left(n+n_{2}\right) \lambda_{1}^{n_{2}}\right)$. Therefore we can consider

$$
\psi_{n}^{(3)}(\lambda)=\sum_{\omega-n_{1} \ldots \omega_{n+n_{1}}} \exp \left\{i \frac{\lambda}{\sqrt{\sigma_{h} \cdot n}} \sum_{s=1}^{p} \sum_{k \in \Delta \zeta^{(1)}} h_{n_{1}}\left(T^{k} \omega\right)\right\} \cdot \mu_{0}\left(\omega_{-n_{1}} \ldots \omega_{n+n_{1}}\right) .
$$

The next step is to replace the probability distribution $\mu_{0}$ by the Markov probability distribution $\mu_{0}^{(1)}$ constructed in Sect. 2 and to consider

$$
\psi_{n}^{(4)}(\lambda)=\sum \exp \left\{i \frac{\lambda}{\sqrt{\sigma_{h} \cdot n}} \sum_{s=1}^{p} \sum_{k \in \Delta \xi_{s}^{(1)}} h_{n_{1}}\left(T^{k} \omega\right)\right\} \mu_{0}^{(1)}\left(\omega_{-n_{1}} \ldots \omega_{n+n_{1}}\right) .
$$

It follows easily from Sect. 2 that the absolute value $\left|\psi_{n}^{(4)}(\lambda)-\psi_{n}^{(3)}(\lambda)\right| \rightarrow 0$ as $n \rightarrow \infty$ uniformly in $\lambda$. Now we can write for $m=n-\left[n^{\gamma_{1}}\right]-\left[n^{\gamma_{2}}\right]$

$$
\begin{aligned}
\psi_{n}^{(4)}(\lambda)= & \sum \exp \left\{i \frac{\lambda}{\sqrt{\sigma_{h} \cdot n}} \sum_{s=1}^{p-1} \sum_{k \in \Delta \xi^{(1)}} h_{n_{1}}\left(T^{k} \omega\right)\right\} \\
& \cdot \mu_{0}^{(1)}\left(\omega_{-n_{1}} \ldots \omega_{m+n_{1}}\right) \cdot \sum_{\omega_{j}, n-[n]^{\gamma_{1}}-n_{1} \leqq j \leqq n+n_{1}} \\
& \cdot \exp \left\{i \frac{\lambda}{\sqrt{\sigma_{h} \cdot n}} \sum_{k \in \Delta \Delta_{p}^{(1)}} h_{n_{1}}\left(T^{k} \omega\right)\right\} \\
& \cdot \mu_{0}^{(1)}\left(\omega_{n-\left[n^{\gamma_{1}}\right]-n_{1}} \ldots \omega_{n+n_{1}} \mid \omega_{m-n_{1}} \ldots \omega_{m+n_{1}}\right) .
\end{aligned}
$$


The same arguments as in Sect. 2 show that the last conditional probabilities depend very weakly on conditions. More precisely,

$$
\begin{aligned}
\mid \sum \exp & \left\{i \frac{\lambda}{\sqrt{\sigma_{h} \cdot n}} \sum_{k \in \Delta_{p}^{(1)}} h_{n_{1}}\left(T^{k} \omega\right)\right\} \\
& \cdot \mu_{0}^{(1)}\left(\omega_{n-\left[n^{\left.\gamma_{1}\right]}-n_{1}\right.} \ldots \omega_{n+n_{1}} \mid \omega_{m-n_{1}} \ldots \omega_{n_{1}+m}\right) \\
& -\sum \exp \left\{i \frac{\lambda}{\sqrt{\sigma_{h} \cdot n}} \sum_{k \in \Delta} h_{n_{1}}\left(T^{k} \omega\right)\right\} \mu_{0}^{(1)}\left(\omega_{n-\left[n^{\gamma_{1}}\right]-n_{1}} \ldots \omega_{n+n_{1}}\right) \mid \\
\leqq & \left(\lambda_{3}^{\prime}\right)^{\left[n^{\gamma_{1}}\right] / n_{1}}+n^{2} \mu_{0}\left(\bar{V}_{n_{1}}\right) .
\end{aligned}
$$

Also the absolute value of the difference

$$
\begin{aligned}
& \sum \exp \left\{i \frac{\lambda}{\sqrt{\sigma_{h} \cdot n}} \sum_{k \in \Delta_{p}^{(1)}} h_{n_{1}}\left(T^{k} \omega\right)\right\} \mu_{0}\left(\omega_{n-\left[n^{\nu_{1}}\right]-n_{1}} \ldots \omega_{n+n_{1}}\right) \\
& -\sum \exp \left\{i \frac{\lambda}{\sqrt{\sigma_{h} \cdot n}} \sum_{\left.k \in \Delta \hat{p}^{1}\right)} h_{n_{1}}\left(T^{k} \omega\right)\right\} \mu_{0}\left(\omega_{n-\left[n^{\gamma_{1}}\right]-n_{1}} \ldots \omega_{n+n_{1}}\right)
\end{aligned}
$$

is sufficiently small. We get as a result that the difference

$$
\left|\psi_{n}^{(4)}(\lambda)-\prod_{r=1}^{p} E \exp \left\{i \frac{\lambda}{\sqrt{\sigma_{h} \cdot n}} \sum_{k \in \Delta_{r}^{(1)}} h_{n_{1}}\left(T^{k} \omega\right)\right\}\right|
$$

tends to zero uniformly in $\lambda$.

It is easy to see that the variance $E\left(\sum_{k \in \Delta_{1}^{(1)}} h_{n_{1}}\left(T^{k} \omega\right)\right)^{2} \sim \sigma_{h} h^{\gamma_{1}}$ and

$$
E\left(\sum_{s=1}^{p} \sum_{k \in \Delta S^{(1)}} h_{n_{1}}\left(T^{k} \omega\right)\right)^{2} \sim \sigma_{h} \cdot n_{1} \cdot p \sim \sigma_{h} \cdot n
$$

for $n \rightarrow \infty$. Thus in order to show the desired limit relation we must check the Lindeberg's condition (see [4]). In view of Chebyshev's inequality it is sufficient to estimate

$$
\begin{aligned}
& E\left(\sum_{k \in \Delta\left(\Delta^{(1)}\right.} h_{n_{1}}\left(T^{k} \omega\right)\right)^{4} \\
& \quad=\sum_{k_{1}, k_{2}, k_{3}, k_{4} \in \Delta(1)} E\left(h_{n_{1}}\left(T^{k_{1}} \omega\right) h_{n_{1}}\left(T^{k_{2}} \omega\right) h_{n_{1}}\left(T^{k_{3}} \omega\right) h_{n_{1}}\left(T^{k_{4}} \omega\right),\right.
\end{aligned}
$$

which can be estimated by const $\left|\Delta_{s}^{(1)}\right|^{2}$ in view of Theorem 1'. Q.E.D.

Now we can formulate a natural extension of Lemma 5.

Lemma 6. Suppose that we have $r$ functions $\left\{h_{1}, \ldots, h_{r}\right\}=h$ with the same properties as in the beginning of Sect. 2 and $E h_{i}=0$ for all $i=1, \ldots, r$. Suppose also that the series

$$
\sum_{n=0}^{\infty} E\left(h_{i}\left(T^{n} \omega\right) \cdot h_{j}(\omega)\right)=\sigma_{i j}(h)
$$

are such that the matrix $\sigma_{h}=\left\|\sigma_{i j}(h)\right\|$ is the positively-definite matrix. Then

$$
\lim _{n \rightarrow \infty} \mu_{0}\left(\omega: a_{i}<\frac{\sum_{k=0}^{n-1} h_{i}\left(T^{k} \omega\right)}{\sqrt{n}}<b_{i}, 1 \leqq i \leqq r\right)=\int_{A} g_{\sigma}\left(u_{1}, \ldots, u_{r}\right) d u
$$


where $A=\left\{u: a_{i}<u_{i}<b_{i}, 1 \leqq i \leqq r\right\} \subset R^{r}, g_{\sigma}$ is the density of the gaussian probability distribution with the covariance matrix equal to $\sigma_{h}$.

The proof of Lemma 6 goes in the same way as the proof of Lemma 5. Therefore we omit the details.

Now we shall derive a weaker version of Theorem 2. Let us take $x_{0} \in \mathscr{M}_{1}$, $x_{1}=T x_{0}$ and put $h_{1}\left(x_{0}\right)=q^{(1)}\left(x_{1}\right)-q^{(1)}\left(x_{0}\right), h_{2}\left(x_{0}\right)=q^{(2)}\left(x_{1}\right)-q^{(2)}\left(x_{0}\right)$. It is easy to see that $E h_{1}=E h_{2}=0$ and $h_{1}, h_{2}$ satisfy the properties described in the beginning of Sect. 2. Thus in order to apply Lemma 6 we must check whether the matrix $\sigma_{h}$, $h=\left(h_{1}, h_{2}\right)$ is non-degenerate.

Suppose that this is wrong. It means that one can find real numbers $a_{1}, a_{2}$ and a function $H(x)$ on the phase space $\mathscr{M}_{1}$ of the billiard problem under consideration for which

$$
a_{1} h_{1}(x)+a_{2} h_{2}(x)=H(T x)-H(x) .
$$

From this equality we have for arbitrary $n$

$$
\sum_{k=0}^{n-1}\left(a_{1} h_{1}\left(T^{k} x\right)+a_{2} h_{2}\left(T^{k} x\right)\right)=H\left(T^{n} x\right)-H(x) .
$$

We shall denote by $\Pi(k)$ a connected rectangular of the plane $R^{2}$ consisting on $k^{2}$ rectangulars i.e.

$$
\Pi(k)=\left\{q=\left(q_{1}, q_{2}\right) \mid 0 \leqq q_{1} \leqq k B_{1}, 0 \leqq q_{2} \leqq k B_{2}\right\} .
$$

Let us consider now a billiard in $\left(\Pi(k) x \times S^{1}\right) \cap \mathscr{M}$. It follows from the equality (2) that for every $\varepsilon>0$ there exists a constant $C_{4}$ not depending on $k$ such that for an arbitrary $n$ the inequality

$$
\left|a_{1} q^{(1)}\left(T^{n} x\right)+a_{2} q^{(2)}\left(T^{n} x\right)\right| \leqq C_{4}
$$

holds with probability (calculated with the help of measure $\mu_{0}$ ) more than $1-\varepsilon$. But from the theory of dispersed billiards it follows that for any $k$ the billiard dynamical system in $\Pi(k)$ is ergodic. It means that for any $n$ the measure $\mu_{k}$ of the set consisting of all points $x$ satisfying the inequality (3) tends to zero as $k \rightarrow \infty$ where $\mu_{k}$ is the invariant measure for billiard in $\Pi(k)$ which is absolutely continuous with respect to Lebesque measure. From this we obtain that $\mu_{0}$-measure of the set of points $x$ satisfying (3) also tends to zero as $k \rightarrow \infty$. Consequently we get a contradiction with the assumption that inequality (3) holds with big probability. Thus the matrix $\sigma_{h}$ is non-degenerate. Q.E.D.

Now from Lemma 6 we derive immediately.

Theorem $2^{\prime}$. Let $q_{k}^{(1)}(x), q_{k}^{(2)}(x)$ be coordinates of the moving point on the plane $R^{2}$ after $k$ reflections from the scatterers. Then

$$
\begin{gathered}
\mu_{0}\left(x: b_{1} \leqq \frac{q_{k}^{(1)}(x)}{\sqrt{k}} \leqq d_{1}, b_{2} \leqq \frac{q_{k}^{(2)}(x)}{\sqrt{k}} \leqq d_{2}\right) \rightarrow \int_{b_{1}}^{d_{1}} \int_{b_{2}}^{d_{2}} g_{\sigma}\left(u_{1}, u_{2}\right) d u_{1} d u_{2} \\
k \rightarrow \infty,
\end{gathered}
$$

where $g_{\sigma}$ is a two-dimensional non-degenerate gaussian probability density. 
Now we proceed to the proof of Theorem 2. Let $x \in \mathscr{M}_{1}$ and $F(x)$ be equal to the time of the motion of this point till the next collision. Then again the function $F\left(\varphi^{-1}(\omega)\right)$ satisfies the conditions of the beginning of Sect. 2 .

Let us denote by $t^{-}$for every $x \in \mathscr{M}$ and every $t>0$ the largest non-positive number, for which $x^{-}=S_{t^{-}}=x \in \mathscr{M}_{1}$ and $t^{+}>t$ be the last number, for which $S_{t^{+}} x$ $=x^{+} \in \mathscr{M}_{1}$. Let $k_{0}=\left[\frac{t}{E F}\right]$ and $k_{1}$ be such that $x^{+}=T^{k_{1}} x$. We can write

$$
\begin{aligned}
\frac{1}{\sqrt{t}} q^{(1)}\left(S_{t} x\right)= & \sqrt{\frac{\sum_{i=0}^{k_{0}-1} F\left(T^{i} x^{-}\right)}{t}} \frac{1}{\sqrt{\sum_{i=0}^{k_{0}-1} F\left(T^{i} x^{-}\right)}} \\
& \cdot \sum_{i=0}^{k_{1}-1} h_{1}\left(T^{i} x^{-}\right)+O\left(\frac{1}{\sqrt{t}}\right)
\end{aligned}
$$

The same formula is true for $\frac{1}{\sqrt{t}} q^{(2)}\left(S_{t} x\right)$. It follows from the usual Birkhoff's ergodic theorem that $\frac{1}{t} \sum_{i=0}^{k_{0}-1} F\left(T^{i} x^{-}\right)$converges in probability to 1 . Therefore

$$
\begin{aligned}
\frac{1}{\sqrt{t}} q^{(1)}\left(S_{t} x\right)= & \frac{1}{\sqrt{\sum_{i=0}^{k_{0}-1} F\left(T^{i} x^{-}\right)}} \sum_{i=0}^{k_{0}-1} h_{1}\left(T^{i} x^{-}\right) \\
& +\frac{1}{\sqrt{\sum_{i=0}^{k_{0}-1} F\left(T^{i} x\right)}} \sum_{i}^{\prime} h_{1}\left(T^{i} x^{-}\right)+\alpha_{t}(x),
\end{aligned}
$$

where $\alpha_{t}(x)$ converges in probability to zero when $t \rightarrow \infty$ and $\Sigma^{\prime}$ is taken over $i$ lying between $k_{0}$ and $k_{1}$. We shall show that

$$
\frac{1}{\sqrt{\sum_{i=0}^{k_{0}-1} F\left(T^{i} x\right)}} \sum_{i}^{\prime} h_{1}\left(T^{i} x^{-}\right)
$$

converges in probability to zero. Let us fix $\varepsilon>0$ and choose $A=A(\varepsilon)$ in such a way that $\mu_{0}\left(\left|k_{0}-k_{1}\right| \geqq A \sqrt{k_{0}}\right) \leqq \varepsilon$. It can be done in view of Lemma 5 . Now the desired result will follow from the assertion that both

$$
\max _{1 \leqq l \leqq A \sqrt{k_{0}}} \frac{1}{\sqrt{k_{0}}}\left|\sum_{k_{0} \leqq i<l+k_{0}} h_{1}\left(T^{i} x^{-}\right)\right|
$$

and

$$
\max _{1 \leqq l \leqq A \sqrt{k_{0}}} \frac{1}{\sqrt{k_{0}}}\left|\sum_{k_{0}-l<i \leqq k_{0}} h_{1}\left(T^{i} x^{-}\right)\right|
$$


converge in probability to zero. Because $T$ is measure preserving we can consider

$$
\max _{1 \leqq l \leqq A \sqrt{k_{0}}} \frac{1}{\sqrt{k_{0}}} \sum_{0 \leqq i \leqq l} h_{1}\left(T^{i} x\right)
$$

and

$$
\max _{1 \leqq l \leqq A \sqrt{k_{0}}} \frac{1}{\sqrt{k_{0}}} \sum_{1 \leqq l \leqq A \sqrt{k_{0}}} h_{1}\left(T^{-i} x\right)
$$

The convergence in probability of last expression to zero is an easy consequence of Birkhoff's ergodic Theorem. Thus we see that the limit of

$$
\mu_{0}\left(x: b_{1} \leqq \frac{1}{\sqrt{t}} q^{(1)}\left(S_{t} x\right) \leqq d_{1}, b_{2} \leqq \frac{1}{\sqrt{t}} q^{(2)}\left(S_{t} x\right) \leqq d_{2}\right)
$$

is the same as the limit of

$$
\begin{aligned}
\mu_{0}\left(x^{-}: b_{1}\right. & \leqq \frac{1}{\sqrt{\sum_{i=0}^{k_{0}-1} F\left(T^{i} x^{-}\right)}} \sum_{i=0}^{k_{0}-1} h_{1}\left(T^{i} x^{-}\right) \leqq d_{1}, \\
b_{2} & \left.\leqq \frac{1}{\sqrt{\sum_{i=0}^{k_{0}-1} F\left(T^{i} x^{-}\right)}} \sum_{i=0}^{k_{0}-1} h_{2}\left(T^{i} x^{-}\right) \leqq d_{2}\right),
\end{aligned}
$$

which in view of Lemma 6 is equal to

$$
\int_{b_{1}}^{d_{1}} \int_{b_{2}}^{d_{2}} g_{\sigma}\left(u_{1}, u_{2}\right) d u_{1} d u_{2}
$$

where $g_{\sigma}$ is the corresponding gaussian density. Q.E.D.

Now we can formulate a direct generalization of Theorem 2.

Theorem 2". Let be chosen $0<s_{1}<s_{2}<\ldots<s_{r}=1$. For every $t>0$ consider random variables $\mathbf{q}\left(s_{i} t\right)=\left(\mathbf{q}^{(1)}\left(s_{i} t\right), q^{(2)}\left(s_{i} t\right)\right), 1 \leqq i \leqq r$, and the normed ones $\frac{1}{\sqrt{t}} \mathbf{q}\left(s_{i} t\right)$. Then for arbitrary pairs of real numbers $b_{i}, d_{i}, b_{i}<d_{i}, 1 \leqq i \leqq r$,

$$
\begin{aligned}
\lim _{t \rightarrow \infty} & \mu_{0}\left(x: b_{i} \leqq \frac{1}{\sqrt{t}} \mathbf{q}\left(s_{i} t\right) \leqq d_{i}, 1 \leqq i \leqq r\right) \\
= & \int_{b_{1}}^{d_{1}} \int_{b_{2}}^{d_{2}} \ldots \int_{b_{r}}^{d_{r}} g_{\sigma}\left(\frac{u_{1}^{(1)}}{\sqrt{s_{1}}}, \frac{u_{2}^{(1)}}{\sqrt{s_{1}}}\right) \cdot g_{\sigma}\left(\frac{u_{1}^{(2)}-u_{1}^{(1)}}{\sqrt{s_{2}-s_{1}}}, \frac{u_{2}^{(2)}-u_{2}^{(1)}}{\sqrt{s_{2}-s_{1}}}\right), \\
& \cdot g_{\sigma}\left(\frac{u_{1}^{(r)}-u_{1}^{(r-1)}}{\sqrt{s_{r}-s_{r-1}}}, \frac{u_{2}^{(r)}-u_{2}^{(r-1)}}{\sqrt{s_{r}-s_{r-1}}}\right) \prod_{i=1}^{r} \prod_{j=1}^{2} d u_{i}^{(j)} .
\end{aligned}
$$




\section{Proof of Theorem 3}

Let $P_{t}$ be a probability distribution on trajectories $\mathbf{q}_{t}(s)=\frac{1}{\sqrt{t}} \mathbf{q}(s t), \quad \mathbf{q}(s t)$ $=\left\{q^{(1)}(s t), q^{(2)}(s t)\right\}, 0 \leqq s \leqq 1$, induced by the probability measure $\mu_{0}$. In view of Theorem 2" and Theorem 8.1 from [5] it is sufficient to show that $P_{t}$ is a tight family of probability measures on the metric space $C_{[0,1]}\left(R^{2}\right)$ of continuous functions $\mathbf{q}(s), 0 \leqq s \leqq 1, \mathbf{q}(s) \in R^{2}$ (see [5]). To do this we shall use Prohorov's theorem [6], [5] which gives the necessary and sufficient condition of tightness of a family of probability distributions. Namely, for every $\varepsilon>0$ there must exist a compact set $K_{\varepsilon}$ in the space $C_{[0,1]}\left(R^{2}\right)$ such that $P_{t}\left(K_{\varepsilon}\right)>1-\varepsilon$ for all $t$.

Using Theorem 8.3 from [5] [more precisely, formula (8.12)] it is sufficient to show that for arbitrary $\varepsilon>0, \eta>0$ there exist $\delta, 0<\delta<1$, and $t_{0}$ such that for all $t \geqq t_{0}$ and all $s, 0 \leqq s \leqq 1$,

$$
\delta^{-1} P_{t}\left\{x: \sup _{s \leqq s^{\prime} \leqq s+\delta}\left\|q_{t}\left(s^{\prime}\right)-q_{t}(s)\right\| \geqq \varepsilon\right\} \leqq \eta .
$$

Thus we fix $\varepsilon, \eta$ and put $\delta \leqq \varepsilon^{7 / 4}$. The value of $t_{0}$ will be chosen during the proof. We decompose the whole segment $[0,1]$ on subintervals by points $0=s_{0}<s_{1}$ $<\ldots<s_{r}=1$ where $s_{i+1}-s_{i} \sim \frac{1}{6} t^{-7 / 12}$ and $\left|s_{i+1}-s_{i}\right| \leqq \frac{1}{3} t^{-7 / 12}$. For each $s, s^{\prime}, 0 \leqq s$, $s^{\prime} \leqq 1$ we take $s_{j_{1}}, s_{j_{2}}$ which are the closest points to $s, s^{\prime}$ and

$$
\begin{aligned}
\left\|q_{t}(s)-q_{t}\left(s^{\prime}\right)\right\| \leqq & \left\|q_{t}\left(s_{j_{1}}\right)-q_{t}\left(s_{j_{2}}\right)\right\| \\
& +\left\|q_{t}\left(s_{j_{1}}\right)-q_{t}(s)\right\|+\left\|q_{t}\left(s_{j_{2}}\right)-q_{t}\left(s^{\prime}\right)\right\| \\
\leqq & \frac{2}{3} \varepsilon+\left\|q_{t}\left(s_{j_{1}}\right)-q_{t}\left(s_{j_{2}}\right)\right\| .
\end{aligned}
$$

Here we have used

$$
\begin{aligned}
\left\|q_{t}\left(s_{1}\right)-q_{t}\left(s_{2}\right)\right\| & =\frac{1}{\sqrt{t}}\left\|q\left(s_{1} t\right)-q\left(s_{2} t\right)\right\| \\
& \leqq \sqrt{t}\left|s_{1}-s_{2}\right| \leqq \frac{1}{3} t^{-1 / 12} \leqq \frac{\varepsilon}{3},
\end{aligned}
$$

if $t$ is large enough. Now in (4) we can consider $s_{j}, s_{j_{1}}$ instead of $s, s^{\prime}$ and estimate the probabilities

$$
P_{t}\left\{x: \max _{s_{j} \leqq s_{j_{1}} \leqq s_{j}+\delta}\left\|q_{t}\left(s_{j}\right)-q_{t}\left(s_{j_{1}}\right)\right\| \geqq \frac{\varepsilon}{3}\right\}
$$

For every $s_{j}$ we put $k_{j}=\frac{t \cdot s_{j}}{E(F(x))}$ and consider the norm of the difference

$$
\left\|q_{t}\left(s_{j}\right)-\frac{1}{\sqrt{t}} \sum_{i=0}^{k_{j}-1} h\left(T^{i} x\right)\right\|=\alpha_{j}(t)
$$

where

$$
h(x)=\left(h^{(1)}(x), h^{(2)}(x)\right)=\left(q^{(1)}(\operatorname{Tx})-q^{(1)}(x), q^{(2)}(\operatorname{Tx})-q^{(2)}(x)\right) .
$$


Lemma 7. $\max _{0 \leqq j \leqq r} \alpha_{j}^{(t)}$ converges in probability to zero as $t \rightarrow \infty$.

Proof of Lemma 7 will be given slightly later. We have now for arbitrary $s_{j}, s_{j_{1}}$

$$
\left\|q_{t}\left(s_{j}\right)-q_{t}\left(s_{j_{1}}\right)\right\| \leqq \frac{1}{\sqrt{t}}\left\|\sum_{k_{J} \leqq i \leqq k_{j_{1}}} \mathbf{h}\left(T^{i} x\right)\right\|+\alpha_{j}^{(t)}+\alpha_{j_{1}}^{(t)} .
$$

If $t$ is large enough, then $P_{t}$-probabilities of the inequalities $\alpha_{j}^{(t)} \leqq \frac{\varepsilon}{12}, \alpha_{j_{1}}^{(t)} \leqq \frac{\varepsilon}{12}$ will be more than $1-\frac{1}{2} \delta \cdot \eta$. Therefore we must estimate only

$$
P_{t}=P_{t}\left\{x: \max _{j_{1}}\left\|\sum_{k_{j} \leqq i \leqq k_{j_{1}}} \mathbf{h}\left(T^{i} x\right)\right\| \geqq \frac{\varepsilon}{6} \sqrt{t}\right\},
$$

where $j \leqq j_{1} \leqq j+\frac{\delta t}{E F(x)}$.

The last probability does not depend on $k_{j}$ because $T$ is measure-preserving. Thus it is sufficient to estimate

$$
\begin{gathered}
P\left\{\max _{0 \leqq k \leqq \delta t / E F}\left|\sum_{0 \leqq i \leqq k} h^{(1)}\left(T^{i} x\right)\right| \geqq \frac{\varepsilon}{12} \sqrt{t}\right\} \\
+P\left\{\max _{0 \leqq k \leqq \delta t / E F}\left|\sum_{0 \leqq i \leqq k} h^{(2)}\left(T^{i} x\right)\right| \geqq \frac{\varepsilon}{12} \sqrt{t}\right\} .
\end{gathered}
$$

Our arguments will be of the same nature as in the proof of the classical Kolmogorov's inequality in the theory of probabilities. We shall consider only the first term. Let be

$$
S_{k}=\left\{x:\left|\sum_{0 \leqq i \leqq j} h^{(1)}\left(T^{i} x\right)\right|<\frac{\varepsilon}{12} \sqrt{t}, j<k \text { and }\left|\sum_{0 \leqq i \leqq k} h^{(1)}\left(T^{i} x\right)\right| \geqq \frac{\varepsilon}{12} \sqrt{t}\right\} .
$$

The subsets $S_{k}$ do not intersect and we are interested in $P=\sum_{0 \leqq k \leqq \delta t / E F} P\left(S_{k}\right)$. We have

$$
\begin{aligned}
P= & \sum_{k} P\left(S_{k}\right) \leqq \frac{12^{4}}{\varepsilon^{4} t^{2}} \sum_{k} \int_{S_{k}}\left[\sum_{0 \leqq i \leqq k} h^{(1)}\left(T^{i} x\right)\right]^{4} d \mu_{0} \\
\leqq & \frac{12^{4}}{\varepsilon^{4} t^{2}} \sum_{k} \int_{S_{k}}\left[\sum_{0 \leqq i \leqq \delta t / E F} h^{(1)}\left(T^{i} x\right)\right]^{4} d \mu_{0}-\frac{4 \cdot 12^{4}}{\varepsilon^{4} t^{2}} \sum_{k} \int_{S_{k}}\left[\sum_{0 \leqq i \leqq k} h^{(1)}\left(T^{i} x\right)\right] \\
& \times\left[\sum_{k<i \leqq \delta t / E F} h^{(1)}\left(T^{i} x\right)\right]^{3} d \mu_{0}-\frac{4 \cdot 12^{4}}{\varepsilon^{4} t^{2}} \sum_{k} \int_{S_{k}}\left[\sum_{0 \leqq i \leqq k} h^{(1)}\left(T^{i} x\right)\right]^{3}\left[\sum_{k<i \leqq \delta t / E F} h^{(1)}\left(T^{i} x\right)\right] d \mu_{0} \\
\leqq & \frac{12^{4}}{\varepsilon^{4} t^{2}} \int\left[\sum_{0 \leqq i \leqq \delta t / E F} h^{(1)}\left(T^{i} x\right)\right]^{4} d \mu_{0}-\frac{4 \cdot 12^{4}}{\varepsilon^{4} t^{2}} \sum_{k} \int_{S_{k}\left[\sum_{0 \leqq i \leqq k} h^{(1)}\left(T^{i} x\right)\right]} \\
& \times\left[\sum_{k<i \leqq \delta t / E F} h^{(1)}\left(T^{i} x\right)\right]^{3} d \mu_{0}-\frac{4 \cdot 12^{4}}{\varepsilon^{4} t^{2}} \sum_{k} \int_{S_{k}}\left[\sum_{0 \leqq i \leqq k} h^{(1)}\left(T^{i} x\right)\right]^{3}\left[\sum_{k<i \leqq \delta t / E F} h^{(1)}\left(T^{i} x\right)\right] d \mu_{0} .
\end{aligned}
$$


The first term is not more than const $\varepsilon^{-4} t^{-2} \delta^{2} t^{2}=\delta$ const $\varepsilon^{-4} \delta$. Suppose that $\delta$ is chosen in such a way that const $\varepsilon^{-4} \delta \leqq \eta$. Then the first term is not more than $\delta \eta$. Thus we must estimate

$$
\begin{aligned}
& I_{1}=\frac{1}{\varepsilon^{4} t^{2}} \sum_{k} \int_{S_{k}}\left[\sum_{0 \leqq i \leqq k} h^{(1)}\left(T^{i} x\right)\right]^{3}\left[\sum_{k<i \leqq \delta t / E F} h^{(1)}\left(T^{i} x\right)\right] d \mu_{0} \\
& I_{2}=\frac{1}{\varepsilon^{4} t^{2}} \sum_{k} \int_{S_{k}}\left[\sum_{0 \leqq i \leqq k} h^{(1)}\left(T^{i} x\right)\right] \cdot\left[\sum_{k<i \leqq \delta t / E F} h^{(1)}\left(T^{i} x\right)\right]^{3} d \mu_{0} .
\end{aligned}
$$

We approximate $h^{(1)}$ by $h_{n_{1}}^{(1)}$ where $n_{1}=\left[n^{\alpha}\right]$ and $\alpha$ is small enough. The error is of order const $t^{\alpha_{1}} \exp \left\{-t^{\alpha_{2}}\right\}, \alpha_{1}, \alpha_{2}>0$ and therefore sufficiently small in order to be neglected. Thus we shall consider

$$
I_{1}^{\prime}=\frac{1}{\varepsilon^{4} t^{2}} \sum_{k} \int_{S_{k}}\left[\sum_{0 \leqq i \leqq k} h_{n_{1}}^{(1)}\left(T^{i} x\right)\right]^{3}\left[\sum_{k<i \leqq \delta t / E F} h_{n_{1}}^{(1)}\left(T^{i} x\right)\right] d \mu_{0} .
$$

For $x \in S_{k}$ we have $\left|\sum_{0 \leqq i \leqq k} h_{n_{1}}^{(1)}\left(T^{i} x\right)\right|^{3} \leqq$ const $\varepsilon^{3} t^{3 / 2}$. The expectation $E h_{n_{1}}^{(1)}=0$. The same arguments as in Sect. 3 show that the conditional expectation of $h_{n_{1}}^{(1)}\left(T^{i} x\right)$ for $i \geqq k+n_{1}^{\alpha_{2}}, \alpha_{2}>1$ when $\omega_{j},-n_{1} \leqq j \leqq k+n_{1}$, are fixed is less than $\left(\lambda_{4}\right)^{\text {const } \frac{i-k}{n_{1}}}$. The part of the sum corresponding to $i, k \leqq i \leqq k+n_{1}^{\alpha_{2}}$, can be estimated simply by const $n_{1}^{\alpha_{2}}$. Thus we get

$$
\begin{aligned}
\left|I_{1}^{\prime}\right| & \leqq \frac{1}{\varepsilon^{4} t^{2}} \sum_{k} \operatorname{const} \varepsilon^{3} t^{3 / 2}\left|\int_{S_{k}}\left[\sum_{k<i \leqq \delta t / E F} h^{(1)}\left(T^{i} x\right)\right] d \mu_{0}\right| \\
& \leqq \frac{\operatorname{const} n_{1}^{\alpha_{2}}}{\varepsilon \sqrt{t}} P .
\end{aligned}
$$

The factor $\frac{n_{1}^{\alpha_{2}}}{\varepsilon \sqrt{t}} \rightarrow 0$ as $t \rightarrow \infty$. In the same way one can estimate $\left|I_{2}^{\prime}\right|$. We have $\left|I_{1}\right|+\left|I_{2}\right| \leqq P \cdot \gamma(t)$ where $\gamma(t) \rightarrow 0$ as $t \rightarrow \infty$.

Now we have $P \leqq \frac{\text { const }}{\varepsilon^{4} t^{2}} E\left(\sum_{0 \leqq i \leqq \delta t / E F} h\left(T^{i} x\right)\right)^{4}$. In view of Theorem $1^{\prime}$ (see Sect. 2) the last expression is not more than const $\varepsilon^{-4} \delta^{2}=\delta \cdot \operatorname{const} \varepsilon^{4} \cdot \delta$. If $\delta$ is so small that const $\varepsilon^{4} \delta \leqq \eta$ then we get the desired estimation.

Proof of Lemma 7. We shall estimate the probability

$$
P\left\{x: \frac{1}{\sqrt{t}}\left|q^{(1)}\left(s_{j} t\right)-\sum_{i=0}^{k_{j}-1} h^{(1)}\left(T^{i} x\right)\right|>\varepsilon\right\} \text {. }
$$

Let $j$ be fixed and $t_{j}(x)=\sum_{i=0}^{k_{j}-1} F\left(T^{i} x\right)$. For every $a>0$ from Chebyshev's inequality and Theorem $1^{\prime}$

$$
\begin{gathered}
P\left\{x:\left|t_{j}(x)-s_{j} t\right|>a\right\} \\
=P\left\{x:\left|\sum_{i=0}^{k_{j}-1} F\left(T^{i} x\right)-E F \cdot k_{j}\right|>a\right\} \leqq \frac{\text { const } k_{j}^{2}}{a^{4}} .
\end{gathered}
$$


We take $a=t^{2 / 3}$. The right-hand term is not more than const $t^{-13 / 64} k_{j}$. Therefore

$$
\sum_{i=0}^{k_{j}^{1}(x)} F\left(T^{i} x\right) \leqq S_{j} t \leqq \sum_{i=0}^{k_{j}^{1}(x)+1} F\left(T^{i} x\right) .
$$

The next remark is that if $\left|t_{j}(x)-s_{j} t\right| \leqq a$ then $\left|k_{j}^{\prime}(x)-k_{j}\right| \leqq$ const $a$. Therefore we can write

$$
\begin{aligned}
& \frac{1}{\sqrt{t}}\left|q^{(1)}\left(s_{j} t\right)-\sum_{i=0}^{k_{j}-1} h^{(1)}\left(T^{i} x\right)\right| \\
\leqq & \frac{1}{\sqrt{t}}\left|\sum_{k_{j}^{1}(x) \leqq i \leqq k_{j}} h^{(1)}\left(T^{i} x\right)\right|+\frac{\text { const }}{\sqrt{t}} .
\end{aligned}
$$

The last problem consists in estimating

$$
P\left\{\max _{i:\left|i-k_{j}\right| \leqq \text { const } a}\left|\sum_{j=k_{j}(x)}^{i} h^{(1)}\left(T^{j} x\right)\right|>b\right\}=P
$$

for $b=\varepsilon \sqrt{t}$. Again our arguments will be of the same nature as in the proof of Kolmogorov's classical inequality in probability theory. Let $S_{k}, k \geqq 0$ be a subset consisting of such $x$ for which

$$
\left|\sum_{j=k_{J}}^{k_{j}+i} h_{1}\left(T^{j} x\right)\right| \leqq b, \quad i<k ; \quad\left|\sum_{j=k_{J}}^{k_{J}+k} h_{1}\left(T^{j} x\right)\right|>b .
$$

The events $S_{k}$ are pairwisely disjoint and we shall estimate $P_{1}=\sum_{k=0}^{\text {const } a} P\left(S_{k}\right)$. An analogous sum of $P\left(S_{k}\right)$ with $k<0$ can be estimated in the same way. From the definition of $S_{k}$ we have

$$
\begin{aligned}
P_{1}= & \sum_{k} P\left(S_{k}\right) \leqq \frac{1}{b^{4}} \sum_{k} \int_{S_{k}}\left[\sum_{k_{J} \leqq i \leqq k_{J}+k} h^{(1)}\left(T^{i} x\right)\right]^{4} d \mu_{0} \\
\leqq & \frac{1}{b^{4}} \int\left[\sum_{k_{j} \leqq i \leqq k_{j}+a} h^{(i)}\left(T^{i} x\right)\right]^{4} d \mu_{0} \\
& -\frac{4}{b^{4}} \sum_{k} \int_{S_{k}}\left[\sum_{k_{j} \leqq i \leqq k_{J}+k} h^{(1)}\left(T^{i} x\right)\right]^{3}\left[\sum_{k_{j}+k<j \leqq k_{j}+\text { const } a} h^{(1)}\left(T^{i} x\right)\right] d \mu_{0} \\
& -\frac{4}{b^{4}} \sum_{k} \int_{S_{k}}\left[\sum_{k_{j} \leqq i \leqq k_{j}+k} h^{(1)}\left(T^{i} x\right)\right]\left[\sum_{k_{j}+k<j \leqq k_{j}+\text { const } a} h^{(1)}\left(T^{i} x\right)\right]^{3} d \mu_{0} .
\end{aligned}
$$

The first term is not more than const $a^{2} b^{-4}=$ const $t^{4 / 3} b^{-4}$ (see Theorem $1^{\prime}$ ). In order to estimate the last sums we approximate $h^{(1)}$ by the function $h_{n_{1}}^{(1)}$ where $n_{1}=\left[t^{\alpha}\right]$ and $\alpha$ is small enough. The error is of order const $t^{\alpha_{1}} \exp \left\{-t^{\alpha_{2}}\right\}$ where $\alpha_{1}$, $\alpha_{2}>0$ and therefore very small. We must estimate only

$$
\frac{1}{b^{4}} \mid \sum_{k} \int_{S_{k}}\left[\sum_{k_{\jmath} \leqq i \leqq k_{j}+k} h_{n_{1}}^{(1)}\left(T^{i} x\right)\right]^{3}\left[\sum_{k_{J}+k<i \leqq k_{j}+\text { const } a} h_{n_{1}}^{(1)}\left(T^{i} x\right)\right] d \mu_{0} .
$$


The first remark is that $\left|\sum_{k_{j} \leqq i \leqq k_{j}+k} \mathrm{~h}_{n_{1}}^{(1)}\left(\mathrm{T}^{i} \mathrm{x}\right)\right|^{3} \leqq(b+\text { const })^{3}$ in view of the definition $S_{k}$. In particular $(b+\text { const })^{3} \leqq$ const $b^{3}$ for $b>1$. The expectation $E h_{n_{1}}^{(1)}=0$. The same arguments as in $\S 3$ show that the conditional expectation of $h_{n_{1}}^{(1)}\left(T^{i} x\right)$ for $i \geqq k_{j}+k+n_{1}^{\alpha_{2}}, \alpha_{2}>1$ is very small and can be neglected. The estimation of the rest part of the last sum is const $b^{-1} n_{1}^{\alpha_{2}} P_{1}$. Similar arguments can be applied to the expression

$$
\frac{1}{b^{4}} \sum_{k} \int_{S_{k}}\left[\sum_{k_{J} \leqq i \leqq k_{j}+k} h_{n_{1}}^{(1)}\left(T^{i} x\right)\right]\left[\sum_{k_{j}+k<i \leqq k_{J}+\text { const } a} h_{n_{1}}^{(1)}\left(T^{i} x\right)\right]^{3} d \mu_{0} .
$$

Here we must estimate conditional expectations of

$$
h_{n_{1}}^{(1)}\left(T^{i_{1}} x\right) h_{n_{1}}^{(1)}\left(T^{i_{2}} x\right) h_{n_{1}}^{(1)}\left(T^{i_{3}} x\right), \quad k_{j}+k \leqq i_{1} \leqq i_{2} \leqq i_{3}
$$

when $\omega_{-n_{1}} \ldots \omega_{k_{\mathrm{J}}+k+n_{1}}$ are fixed.

These expectations are very small if $i_{3}-i_{2} \geqq n_{1}^{\alpha_{2}}$. The total amount of other terms is less than const $a^{2} n_{1}^{\alpha_{2}}$. Therefore the absolute value of the whole sum is not more than const $b^{-3} a^{2} n_{1}^{\alpha_{2}} P_{1}$. Finally we get for sufficiently small $\alpha_{2}$ and sufficiently large $t$

$$
P_{1} \leqq \text { const } t^{113 / 84} b^{-4}=\text { const } \varepsilon^{-4} t^{-55 / 84}
$$

The whole estimation of $P$ takes an analogous form

$$
P \leqq \text { const } \varepsilon^{-4} t^{-55 / 84}
$$

The final result follows from the last estimation because the total number $N$ of $j$ is not more than const $t^{7 / 12}$ and therefore

$$
\begin{gathered}
P\left\{\max _{j} \max _{i:\left|k_{J}-i\right| \leqq \text { const } a}\left|\frac{1}{\sqrt{t}} \sum h^{(1)}\left(T^{i} x\right)\right| \geqq \varepsilon\right\} \\
\leqq N \cdot P=\mathrm{const} t^{7 / 12} \varepsilon^{-4} t^{-55 / 84}=\mathrm{const} \varepsilon^{-4} t^{-1 / 14} \rightarrow 0
\end{gathered}
$$

as $t \rightarrow \infty$. Q.E.D.

\section{References}

1. Keller, G.: Diplomarbeit, Erlangen-Nürnberg 1977

2. Bunimovich, L.A., Sinai, Ya.G.: Markov partitions for dispersed billiards. Commun. Math. Phys. 77, 247-280 (1980)

3. Doob, J.L.: Stochastic processes. New York: Wiley and Sons 1950

4. Gnedenko, B.V., Kolmogorov, A.N.: Limit theorems for sums of independent random variables. Moscow: Gostehizdat (1947)

5. Billingsley, P.: Convergence of probability measures. New York: Wiley and Sons 1970

6. Prohorov, Ju.V.: Theory of probabilities and its applications, Vol. 1, No. 2, p. 177 (1956)

Communicated by A. Jaffe 
